\title{
Volumetric Transfer of Liquids
}

\author{
Lane C. Sander \\ National Institute of Standards and Technology, \\ Gaithersburg, MD 20899, USA \\ lane.sander@nist.gov
}

Video DOI: http://doi.org/10.18434/T4DK5T

Key words: air displacement pipette; pipette; positive displacement pipette; quantitation; volumetric transfer.

Accepted: December 1, 2016

Published: January 12, 2017

https://doi.org/10.6028/jres.122.002

\section{Summary}

Although volumetric measurements are usually less accurate than gravimetric based measurements, volumetric measurements are still in common use due to the convenience, and often higher throughput, that may be afforded by automatic pipettes. Analysts should be aware of the performance limitations that can be expected for methods that rely on volumetric measurements, and precautions that must be observed to achieve the best results. This presentation highlights the use of different types of automatic pipettes and demonstrates pipetting operations for aqueous and organic liquids. Topics include pipette tip selection, positive displacement and air displacement pipettes, operation of mechanical and electronic pipettes, and performance expectations. ${ }^{1}$

\footnotetext{
${ }^{1}$ Contribution of the National Institute of Standards and Technology. Not subject to copyright. Certain commercial equipment, instruments, or materials are identified to specify adequately the experimental procedure. Such identification does not imply recommendation or endorsement by the National Institute of Standards and Technology, nor does it imply that the materials or equipment identified are the best available for the purpose.
} 